\title{
Gaussian random projections for Euclidean membership problems
}

\author{
Vu Khac Kr11, Pierre-Louis Poirion, Leo LiBerti \\ CNRS LIX, École Polytechnique, F-91128 Palaiseau, France \\ Email vu, poirion, liberti@lix.polytechnique.fr
}

August 2, 2018

\begin{abstract}
We discuss the application of random projections to the fundamental problem of deciding whether a given point in a Euclidean space belongs to a given set. We show that, under a number of different assumptions, the feasibility and infeasibility of this problem are preserved with high probability when the problem data is projected to a lower dimensional space. Our results are applicable to any algorithmic setting which needs to solve Euclidean membership problems in a high-dimensional space.
\end{abstract}

\section{Introduction}

Random projections are very useful dimension reduction techniques which are widely used in computer science [7, 13]. We assume we have an algorithm $\mathcal{A}$ acting on a data set $X$ consisting of $n$ vectors in $\mathbb{R}^{m}$, where $m$ is large, and assume that the complexity of $\mathcal{A}$ depends on $m$ and $n$ in a way that makes it impossible to run $\mathcal{A}$ sufficiently fast. A random projection exploits the statistical properties of some random distribution to construct a mapping which embeds $X$ into a lower dimensional space $\mathbb{R}^{k}$ (for some appropriately chosen $k$ ) while preserving distances, angles, or other quantities used by $\mathcal{A}$.

One striking example of random projections is the famous Johnson-Lindenstrauss lemma [9]:

\subsection{Theorem (Johnson-Lindenstrauss Lemma)}

Let $X$ be a set of $m$ points in $\mathbb{R}^{m}$ and $\varepsilon>0$. Then there is a map $F: \mathbb{R}^{m} \rightarrow \mathbb{R}^{k}$ where $k$ is $O\left(\frac{\log m}{\varepsilon^{2}}\right)$, such that for any $x, y \in X$, we have

$$
(1-\varepsilon)\|x-y\|_{2}^{2} \leq\|F(x)-F(y)\|_{2}^{2} \leq(1+\varepsilon)\|x-y\|_{2}^{2} .
$$

Intuitively, this lemma claims that $X$ can be projected in a much lower dimensional space whilst keeping Euclidean distances approximately the same. The main idea to prove Thm. 1.1 is to construct a random linear mapping $T$ (called $J L$ random mapping onwards), sampled from certain distribution families, so that for each $x \in \mathbb{R}^{m}$, the event that

$$
(1-\varepsilon)\|x\|_{2}^{2} \leq\|T(x)\|_{2}^{2} \leq(1+\varepsilon)\|x\|_{2}^{2}
$$

\footnotetext{
${ }^{1}$ Supported by a Microsft Research Ph.D. fellowship.
} 
occurs with high probability. By Eq. (2) and the union bound, it is possible to show the existence of a map $F$ with the stated properties (see [2, 4]).

In this paper we employ random projections to study the following general problem:

Euclidean Set Membership Problem (ESMP). Given $p \in \mathbb{R}^{m}$ and $X \subseteq \mathbb{R}^{m}$, decide whether $p \in X$.

This is a fundamental class consisting of many problems, both in $\mathbf{P}$ (e.g. the Linear FEASIBILity Problem (LFP)) and NP-hard (e.g. the Integer Feasibility Problem (IFP), which can naturally model SAT, and also see [15]).

In this paper, we use a random linear projection operator $T$ to embed both $p$ and $X$ to a lower dimensional space, and study the relationship between the original membership problem and its projected version:

Projected ESMP (PESMP). Given $p, X, T$ as above, decide whether $T(p) \in T(X)$.

Note that, when $p \in X$, the fact that $T(p) \in T(X)$ follows by linearity of $T$. We are therefore only interested in the case when $p \notin X$, i.e. we want to estimate $\operatorname{Prob}(T(p) \notin T(X))$, given that $p \notin X$.

\subsection{Previous results}

Random projections applying to some special cases of membership problems have been studied in [11, where we exploited some polyhedral structures of the problem to derive several results for polytopes and polyhedral cones. In the case $X$ is a polytope, we obtained the following result.

\subsection{Proposition ([1] $)$}

Given $a_{1}, \ldots, a_{n} \in \mathbb{R}^{m}$, let $C=\operatorname{conv}\left\{a_{1}, \ldots, a_{n}\right\}, b \in \mathbb{R}^{m}$ such that $b \notin C, d=\min _{x \in C}\|b-x\|$ and $D=\max _{1 \leq i \leq n}\left\|b-a_{i}\right\|$. Let $T: \mathbb{R}^{m} \rightarrow \mathbb{R}^{k}$ be a JL random mapping. Then

$$
\operatorname{Prob}(T(b) \notin T(C)) \geq 1-2 n^{2} e^{-\mathcal{C}\left(\varepsilon^{2}-\varepsilon^{3}\right) k}
$$

for some constant $\mathcal{C}$ (independent of $m, n, k, d, D$ ) and $\varepsilon<\frac{d^{2}}{D^{2}}$.

If $X$ is a polyhedral cone, we obtained the following result.

\subsection{Proposition ([1] $)$}

Given $b, a_{1}, \ldots, a_{n} \in \mathbb{R}^{m}$ of norms 1 such that $b \notin C=$ cone $\left\{a_{1}, \ldots, a_{n}\right\}$, let $d=\min _{x \in C}\|b-x\|$ and $T: \mathbb{R}^{m} \rightarrow \mathbb{R}^{k}$ be a JL random mapping. Then:

$$
\operatorname{Prob}(T(b) \notin T(C)) \geq 1-2 n(n+1) e^{-\mathcal{C}\left(\varepsilon^{2}-\varepsilon^{3}\right) k}
$$

for some constant $\mathcal{C}$ (independent of $m, n, k, d$ ), where $\varepsilon=\frac{d^{2}}{\mu_{A}^{2}+2 \sqrt{1-d^{2}} \mu_{A}+1}$,

$$
\mu_{A}=\max \left\{\|x\|_{A} \mid x \in \operatorname{cone}\left(a_{1}, \ldots, a_{n}\right) \wedge\|x\| \leq 1\right\},
$$

and $\|x\|_{A}=\min \left\{\sum_{i} \theta_{i} \mid \theta \geq 0 \wedge x=\sum_{i} \theta_{i} a_{i}\right\}$ is the norm induced by $A=\left(a_{1}, \ldots, a_{n}\right)$. 
We also recall the following Lemma, useful for the integer case.

1.4 Lemma ([11])

Let $T: \mathbb{R}^{m} \rightarrow \mathbb{R}^{k}$ be a $J L$ random mapping, let $b, a_{1}, \ldots, a_{n} \in \mathbb{R}^{m}$ and let $X \subseteq \mathbb{R}^{m}$ be a finite set. Then if $b \neq \sum_{i=1}^{m} y_{i} a_{i}$ for all $y \in X$, we have

$$
\operatorname{Prob}\left(\forall y \in X \mid T(b) \neq \sum_{i=1}^{m} y_{i} T\left(a_{i}\right)\right) \geq 1-2|X| e^{-\mathcal{C} k} ;
$$

for some constant $\mathcal{C}>0$ (independent of $m, k$ ).

\subsection{New results}

In this paper, we consider the general case where the data set $X$ has no specific structure, and use Gaussian random projections in our arguments to obtain some results about the relationship between ESMP and PESMP.

In the case when $X$ is at most countable (i.e. finite or countable), using a straightforward argument, we prove that these two problems are equivalent almost surely. However, this result is only of theoretical interest due to round-off errors in floating point operations, which make its practical application difficult. We address this issue by introducing a threshold $\delta>0$ with a corresponding Threshold ESMP (TESMP): if $\Delta$ is the distance between $T(p)$ and the closest point of $T(X)$, decide whether $\Delta \geq \delta$.

In the case when $X$ may also be uncountable, we employ the doubling constant of $X$, i.e. the smallest number $\lambda_{X}$ such that any closed ball in $X$ can be covered by at most $\lambda_{X}$ closed balls of half the radius. Its logarithm $\log _{2} \lambda_{X}$ is called doubling dimension of $X$. Recently, the doubling dimension has become a powerful tool for several classes of problems such as nearest neighbor [10, 8], low-distortion embeddings [3], clustering [12].

We show that we can project $X$ into $\mathbb{R}^{k}$, where $k=O\left(\log _{2} \lambda_{X}\right)$, whilst still ensure the equivalence between ESMP and PESMP with high probability. We also extend this result to the threshold case, and obtain a more useful bound for $k$.

\section{$2 \quad$ Finite and countable sets}

In this section, we assume that $X$ is either finite or countable. Let $T$ be a JL random mapping from a Gaussian distribution, i.e. each entry of $T$ is independently sampled from $\mathcal{N}(0,1)$. It is well known that, for an arbitrary unit vector $a \in \mathbb{S}^{m-1}$, the random variable $\|T a\|^{2}$ has a Chi-squared distribution $\chi_{k}^{2}$ with $k$ degrees of freedom ([14]). Its corresponding density function is $\frac{2^{-k / 2}}{\Gamma(k / 2)} x^{k / 2-1} e^{k / 2}$, where $\Gamma(\cdot)$ is the gamma function. By [4, for any $0<\delta<1$, taking $z=\frac{\delta}{k}$ yields a cumulative distribution function

$$
F_{\chi_{k}^{2}}(\delta) \leq\left(z e^{1-z}\right)^{k / 2}<(z e)^{k / 2}=\left(\frac{e \delta}{k}\right)^{k / 2}
$$

Thus, we have

$$
\operatorname{Prob}(\|T a\| \leq \delta)=F_{\chi_{k}^{2}}\left(\delta^{2}\right)<\left(3 \delta^{2}\right)^{k / 2}
$$


or, more simply, $\operatorname{Prob}(\|T a\| \leq \delta)<\delta^{k}$ when $k \geq 3$.

Using this estimation, we immediately obtain the following result.

\subsection{Proposition}

Given $p \in \mathbb{R}^{m}$ and $X \subseteq \mathbb{R}^{m}$, at most countable, such that $p \notin X$. Then, for a Gaussian random projection $T: \mathbb{R}^{m} \rightarrow \mathbb{R}^{k}$ with any $k \geq 1$, we have $T(p) \notin T(X)$ almost surely, i.e. $\operatorname{Prob}(T(p) \notin$ $T(X))=1$.

Proof. First, note that for any $u \neq 0, T u \neq 0$ holds almost certainly. Indeed, without loss of generality we can assume that $\|u\|=1$. Then for any $0<\delta<1$ :

$$
\operatorname{Prob}(T(z)=0) \leq \operatorname{Prob}(\|T z\| \leq \delta)=\left(3 \delta^{2}\right)^{k / 2} \rightarrow 0 \text { as } \delta \rightarrow 0 .
$$

Since the event $T(p) \notin T(X)$ can be written as the intersection of at most countably many almost sure events $T(p) \neq T(x)$ (for $x \in X$ ), it follows that $\operatorname{Prob}(T(p) \notin T(X))=1$, as claimed.

Proposition 2.1 is simple, but it looks interesting because it suggests that we only need to project the data points to a line (i.e. $k=1$ ) and study an equivalent membership problem on a line. Furthermore, it turns out that this result remains true for a large class of random projections.

\subsection{Proposition}

Let $\nu$ be a probability distribution on $\mathbb{R}^{m}$ with bounded Lebesgue density $f$. Let $Y \subseteq \mathbb{R}^{m}$ be an at most countable set such that $0 \notin Y$. Then, for a random projection $T: \mathbb{R}^{m} \rightarrow \mathbb{R}^{1}$ sampled from $\nu$, we have $0 \notin T(Y)$ almost surely, i.e. $\operatorname{Prob}(0 \notin T(Y))=1$.

Proof. For any $0 \neq y \in Y$, consider the set $\mathcal{E}_{y}=\left\{T: \mathbb{R}^{m} \rightarrow \mathbb{R}^{1} \mid T(y)=0\right\}$. If we regard each $T: \mathbb{R}^{m} \rightarrow \mathbb{R}^{1}$ as a vector $t \in \mathbb{R}^{m}$, then $\mathcal{E}_{y}$ is a hyperplane $\left\{t \in \mathbb{R}^{m} \mid y \cdot t=0\right\}$ and we have

$$
\operatorname{Prob}(T(y)=0)=\nu\left(\mathcal{E}_{y}\right)=\int_{\mathcal{E}_{y}} f d \mu \leq\|f\|_{\infty} \int_{\mathcal{E}_{y}} d \mu=0
$$

where $\mu$ denotes the Lebesgue measure on $\mathbb{R}^{m}$. The proof then follows by the countability of $Y$, similarly to Proposition 2.1.

Proposition $[2.2$ is based on the observation that the degree $[\mathbb{R}: \mathbb{Q}]$ of the field extension $\mathbb{R} / \mathbb{Q}$ is $2^{\aleph_{0}}$, whereas $Y$ is countable; so the probability that any row vector $T_{i}$ of the random projection matrix $T$ will yield a linear dependence relation $\sum_{j \leq m} T_{i j} y_{j}=0$ for some $0 \neq y \in Y$ is zero. In practice, however, $Y$ is part of the rational input of a decision problem, and the components of $T$ are rational: hence any subsequence of them is trivially linearly dependent over $\mathbb{Q}$. Moreover, floating point numbers have a bounded binary representation: hence, even if $Y$ is finite, there is a nonzero probability that any subsequence of components of $T$ will be linearly dependent by means of a nonzero multiplier vector in $Y$.

This idea, however, does not work in practice: we tested it by considering the ESMP given by the IPF defined on the set $\left\{x \in \mathbb{Z}_{+}^{n} \cap[L, U] \mid A x=b\right\}$. Numerical experiments indicate that the corresponding PESMP $\left\{x \in \mathbb{Z}_{+}^{n} \cap[L, U] \mid T(A) x=T(b)\right\}$, with $T$ consisting of a one-row Gaussian projection matrix, is always feasible despite the infeasibility of the original IPF. Since Prop. 2.1 
assumes that the components of $T$ are real numbers, we think that the reason behind this failure is the round-off error associated to the floating point representation used in computers. Specifically, when $T(A) x$ is too close to $T(b)$, floating point operations will consider them as a single point. In order to address this issue, we force the projected problems to obey stricter requirements. In particular, instead of only requiring that $T(p) \notin T(X)$, we ensure that

$$
\operatorname{dist}(T(p), T(X))=\min _{x \in X}\|T(p)-T(x)\|>\tau,
$$

where dist denotes the Euclidean distance, and $\tau>0$ is a (small) given constant. With this restriction, we obtain the following result.

\subsection{Proposition}

Given $\tau, \delta>0$ and $p \notin X \subseteq \mathbb{R}^{m}$, where $X$ is a finite set, let

$$
d=\min _{x \in X}\|p-x\|>0
$$

Let $T: \mathbb{R}^{m} \rightarrow \mathbb{R}^{k}$ be a Gaussian random projection with $k \geq \frac{\log \left(\frac{|X|}{\delta}\right)}{\log \left(\frac{d}{\tau}\right)}$. Then:

$$
\operatorname{Prob}\left(\min _{x \in X}\|T(p)-T(x)\|>\tau\right)>1-\delta
$$

Proof. We assume that $k \geq 3$. For any $x \in X$ we have:

$$
\begin{aligned}
\operatorname{Prob}(\|T(p-x)\| \leq \tau) & =\operatorname{Prob}\left(\left\|T\left(\frac{p-x}{\|p-x\|}\right)\right\| \leq \frac{\tau}{\|p-x\|}\right) \\
& \leq \operatorname{Prob}\left(\left\|T\left(\frac{p-x}{\|p-x\|}\right)\right\| \leq \frac{\tau}{d}\right)<\frac{\tau^{k}}{d^{k}},
\end{aligned}
$$

due to (3). Therefore, by the union bound,

$$
\begin{aligned}
\operatorname{Prob}\left(\min _{x \in X}\|T(p)-T(x)\|>\tau\right) & =1-\operatorname{Prob}\left(\min _{x \in X}\|T(p)-T(x)\| \leq \tau\right) \\
& \geq 1-\sum_{x \in X} \operatorname{Prob}(\|T(p)-T(x)\| \leq \tau)>1-|X|\left(\frac{\tau}{d}\right)^{k} .
\end{aligned}
$$

The RHS is greater than or equal to $1-\delta$ if and only if $\left(\frac{d}{\tau}\right)^{k} \geq \frac{|X|}{\delta}$, which is equivalent to $k \geq \frac{\log \left(\frac{|X|}{\delta}\right)}{\log \left(\frac{d}{\tau}\right)}$, as claimed.

Note that $d$ is often unknown and can be arbitrarily small. However, if both $p, X$ are integral, then $d \geq 1$ and we can select $k>\frac{\log \frac{|X|}{\delta}}{\log \frac{1}{\tau}}$ in the above proposition.

In many cases, the set $X$ is infinite. We show that when this is the case, we can still overcome this difficulty under some assumptions. In particular, we prove that if $X=\left\{A x \mid x \in \mathbb{Z}_{+}^{n}\right\}$ where $A$ is an $m \times n$ matrix with integer coefficients which are all positive in at least one row, then for any bounded vector $b \in \mathbb{Z}^{m}$ the problem $b \in X$ is equivalent, with high probability, to its projection to a $O(\log n)$-dimensional space. The idea is to separate one positive row and apply random projection to the others. 
Formally, let us denote by $a^{i}$ the $i$-th row and by $a_{j}$ the $j$-th column of $A$. Assume that all entries in the row $a^{i}$ is positive and all entries of $b$ are bounded by a constant $B>0$. Remove the row $i$ from $A$ and $b$ to obtain $\tilde{A}=\left(a_{1}^{\prime}, \ldots, a_{n}^{\prime}\right) \in \mathbb{Z}^{(m-1) \times n}$ and $\tilde{b} \in \mathbb{Z}^{m-1}$. Let $T: \mathbb{R}^{m-1} \rightarrow \mathbb{R}^{k}$ be a JL random mapping and denote by $Z=\left\{x \in \mathbb{Z}_{+}^{n} \mid a^{i} \cdot x=b_{i}\right\}$. Then we have:

\subsection{Proposition}

Assume that $b \notin X$, and let $0<\delta<1$. Using the terminology and given the assumptions above, if $k \geq \frac{1}{\mathcal{C}} \ln \left(\frac{2}{\delta}\right)+\frac{B}{\mathcal{C}} \log (n+B-1)$ we have

$$
\operatorname{Prob}\left(T(b) \neq \sum_{j=1}^{n} x_{j} T\left(a_{j}^{\prime}\right) \text { for all } x \in Z\right) \geq 1-\delta
$$

for some constant $\mathcal{C}>0$.

Proof. We first show that $|Z| \leq(n+B-1)^{B}$. Since all the entries of $A$ are positive integers, we have

$$
|Z| \leq\left|\left\{x \in \mathbb{Z}_{+}^{n} \mid \sum_{j=1}^{n} x_{j}=b_{i}\right\}\right| \leq\left|\left\{x \in \mathbb{Z}_{+}^{n} \mid \sum_{j=1}^{n} x_{j}=B\right\}\right| .
$$

The number of elements in the RHS corresponds to the number of combinations with repetitions of $B$ items sampled from $n$, which is equal to $\left(\begin{array}{c}n+B-1 \\ n-1\end{array}\right)=\left(\begin{array}{c}n+B-1 \\ B\end{array}\right) \leq(n+B-1)^{B}$.

Next, by Lemma 1.4, we have:

$$
\operatorname{Prob}\left(T(b) \neq \sum_{j=1}^{n} x_{j} T\left(a_{j}^{\prime}\right) \text { for all } x \in Z\right) \geq 1-2(n+B-1)^{B} e^{-\mathcal{C} k},
$$

which is greater than $1-\delta$ when taking any $k$ such that $k \geq \frac{1}{\mathcal{C}} \ln \left(\frac{2}{\delta}\right)+\frac{B}{\mathcal{C}} \log (n+B-1)$. The proposition is proved.

Note that in Prop. 2.4 we can choose the JL random mapping $T$ as a matrix with $\{-1,+1\}$ entries (Rademacher variables). In this case, there is no need to worry about floating point errors.

\section{Sets with low doubling dimension}

In this section, we denote by $B(x, r)$ the closed ball centered at $x$ with radius $r>0$, and $B_{X}(x, r)=$ $B(x, r) \cap X$. We will also assume that $X$ is a doubling space, i.e. a set with bounded doubling dimension. One example of doubling spaces is a Euclidean space. $\mathbb{R}^{m}$, we can show that the doubling dimension $\log _{2}\left(\lambda_{X}\right)$ of $X$ can be shown to be a constant factor of $m([16,6])$. However, many sets of low doubling dimensions are contained in high dimensional spaces ([1]). Note that computing the doubling dimension of a metric space is generally NP-hard ([5]). We shall make use of the following simple lemma.

\subsection{Lemma}

For any $p \in X$ and $\varepsilon, r>0$, there is a set $S \subseteq X$ of size at most $\lambda_{X}^{\left\lceil\log _{2}\left(\frac{r}{\varepsilon}\right)\right\rceil}$ such that

$$
B_{X}(p, r) \subseteq \bigcup_{s \in S_{j}} B(s, \varepsilon)
$$


Proof. By definition of the doubling dimension, $B_{X}(p, r)$ is covered by at most $\lambda_{X}$ closed balls of radius $\frac{r}{2}$. Each of these balls in turn is covered by $\lambda_{X}$ balls of radius $\frac{r}{4}$, and so on: iteratively, for each $k \geq 1, B_{X}(p, r)$ is covered by $\lambda_{X}^{k}$ balls of radius $\frac{r}{2^{k}}$. If we select $k=\left\lceil\log _{2}\left(\frac{r}{\varepsilon}\right)\right\rceil$ then $k \geq \log _{2}\left(\frac{r}{\varepsilon}\right)$, i.e. $\frac{r}{2^{k}} \leq \varepsilon$. This means $B_{X}(p, r)$ is covered by $\lambda_{X}^{\left\lceil\log _{2}\left(\frac{r}{\varepsilon}\right)\right\rceil}$ balls of radius $\varepsilon$.

We will also use the following lemma, which is proved in [8] using a concentration estimation for sum of squared gaussian variables (Chi-squared distribution).

\subsection{Lemma}

Let $X \subseteq B(0,1)$ be a subset of the $m$-dimensional Euclidean unit ball. Then there exist universal constants $c, C>0$ such that for $k \geq C \log \lambda_{X}+1$ and $\delta>1$, the following holds:

$$
\operatorname{Prob}(\exists x \in X \text { s.t. }\|T x\|>\delta)<e^{-c k \delta^{2}} .
$$

In the proof of the next result (one of the main results in this section), we use the same idea as that in [8] for the nearest neighbor problem.

\subsection{Theorem}

Given $0<\delta<1$ and $p \notin X \subseteq \mathbb{R}^{m}$. Let $T: \mathbb{R}^{m} \rightarrow \mathbb{R}^{k}$ be a Gaussian random projection. Then

$$
\operatorname{Prob}(T(p) \notin T(X))=1
$$

if $k \geq \mathcal{C} \log _{2}\left(\lambda_{X}\right)$, for some universal constant $\mathcal{C}$.

Proof. Let $\varepsilon>0$ and $0=r_{0}<r_{1}<r_{2}<\ldots$ be positive scalars (their values will be defined later). For each $j=1,2,3, \ldots$ we define a set

$$
X_{j}=X \cap B\left(p, r_{j}\right) \backslash B\left(p, r_{j-1}\right) .
$$

Since $X_{j} \subseteq B_{X}\left(p, r_{j}\right)$, by Lemma 3.1 we can find a point set $S_{j} \subseteq X$ of size $\left|S_{j}\right| \leq \lambda_{X}^{\left\lceil\log _{2}\left(\frac{r_{j}}{\varepsilon}\right)\right\rceil}$ such that

$$
X_{j} \subseteq \bigcup_{s \in S_{j}} B(s, \varepsilon)
$$

Hence, for any $x \in X_{j}$, there is $s \in S_{j}$ such that $\|x-s\|<\varepsilon$. Moreover, by the triangle inequality, any such $s$ satisfies $r_{j-1}-\varepsilon<\|s-p\|<r_{j}+\varepsilon$, so without loss of generality we can assume that

$$
S_{j} \subseteq B\left(p, r_{j}+\varepsilon\right) \backslash B\left(p, r_{j-1}-\varepsilon\right) .
$$

We denote by $\mathcal{E}_{j}$ the event that:

$$
\exists s \in S_{j}, \exists x \in X_{j} \cap B(s, \varepsilon) \text { s.t. }\|T s-T x\|>\varepsilon \sqrt{j} .
$$

By the union bound, we have

$$
\begin{aligned}
\operatorname{Prob}\left(\mathcal{E}_{j}\right) & \leq \sum_{s \in S_{j}} \operatorname{Prob}\left(\exists x \in X_{j} \cap B(s, \varepsilon) \text { s.t. }\|T s-T x\|>\varepsilon \sqrt{j}\right) \\
& \leq \sum_{s \in S_{j}} e^{-c_{1} k j} \quad \text { (for some universal constant } c_{1} \text { by Lemma 3.2) } \\
& \leq \lambda_{X}^{\left\lceil\log _{2}\left(\frac{r_{j}+\varepsilon}{\varepsilon}\right)\right\rceil} e^{-c_{1} k j} .
\end{aligned}
$$


Again by the union bound, we have:

$$
\begin{aligned}
\operatorname{Prob}(\exists x \in X \text { s.t } T(x)=T(p)) & =\operatorname{Prob}\left(\exists x \in \bigcup_{j=1}^{\infty} X_{j} \text { s.t } T(x)=T(p)\right) \\
& \leq \sum_{j=1}^{\infty} \operatorname{Prob}\left(\exists x \in X_{j} \text { s.t } T(x)=T(p)\right)
\end{aligned}
$$

Now we will estimate the individual probabilities:

$$
\begin{aligned}
& \operatorname{Prob}\left(\exists x \in X_{j} \text { s.t } T(x)=T(p)\right) \\
\leq & \operatorname{Prob}\left(\left(\exists x \in X_{j} \text { s.t } T(x)=T(p)\right) \wedge \mathcal{E}_{j}^{c}\right)+\operatorname{Prob}\left(\mathcal{E}_{j}\right) \\
\leq & \operatorname{Prob}\left(\exists x \in X_{j}, s \in S_{j} \cap B(x, \varepsilon) \text { s.t } T(x)=T(p) \wedge\|T(s)-T(x)\| \leq \varepsilon \sqrt{j}\right)+\operatorname{Prob}\left(\mathcal{E}_{j}\right) \\
\leq & \operatorname{Prob}\left(\exists s \in S_{j} \text { s.t }\|T(s)-T(p)\|<\varepsilon \sqrt{j}\right)+\lambda_{X}^{\left\lceil\log _{2}\left(\frac{r_{j}+\varepsilon}{\varepsilon}\right)\right\rceil} e^{-c_{1} k j} .
\end{aligned}
$$

Next, we choose $\varepsilon=\frac{d}{N}$ for some large $N$; and for each $j \geq 1$, we choose $r_{j}=(2+j) \varepsilon$. For $j<N-2$, by definition it follows that $X_{j}=\varnothing$. Therefore

$$
\operatorname{Prob}\left(\exists x \in X_{j} \text { s.t } T(s)=T(p)\right)=0 .
$$

On the other hand, for $j \geq N-2$,

$$
\begin{aligned}
& \operatorname{Prob}\left(\exists s \in S_{j} \text { s.t }\|T(s)-T(p)\| \leq \varepsilon \sqrt{j}\right) \\
\leq & \lambda_{X}^{\left\lceil\log _{2}\left(\frac{r_{j}+\varepsilon}{\varepsilon}\right)\right\rceil} \operatorname{Prob}\left(\|T(z)\| \leq \frac{\varepsilon \sqrt{j}}{r_{j-1}-\varepsilon}\right) \quad \text { for an arbitrary } z \in \mathbb{S}^{n-1} \\
= & \lambda_{X}^{\left\lceil\log _{2}(3+j)\right\rceil} \operatorname{Prob}\left(\|T(z)\| \leq \frac{1}{\sqrt{j}}\right) \quad \text { for an arbitrary } z \in \mathbb{S}^{n-1} \\
< & \lambda_{X}^{\left\lceil\log _{2}(3+j)\right\rceil} j^{-k / 2} \quad \text { by the estimation (4). }
\end{aligned}
$$

Note that $\lambda_{X}^{\left\lceil\log _{2}(3+j)\right\rceil} \leq \lambda_{X}^{\log _{2}(6+2 j)}=(6+2 j)^{\log _{2} \lambda_{X}}<j^{\left(2 \log _{2} \lambda_{X}\right)}$ for large enough $N$. Therefore, we have

$$
\begin{aligned}
\operatorname{Prob}\left(\exists x \in X_{j} \text { s.t } T(x)=T(p)\right) & \leq \lambda_{X}^{\left\lceil\log _{2}(3+j)\right\rceil}\left(j^{-k / 2}+e^{-c_{1} k j}\right) \\
& \leq j^{-c_{2} k}+e^{-c_{3} k j}
\end{aligned}
$$

for some universal constants $c_{2}, c_{3}$, provided that $k \geq \mathcal{C}_{1} \log \lambda_{X}$ for some large enough constant $\mathcal{C}_{1}$. Finally, by the union bound,

$$
\begin{aligned}
\operatorname{Prob}(T(p) \notin T(X)) & =1-\operatorname{Prob}(T(p) \in T(X)) \\
& \geq 1-\sum_{i=N-2}^{\infty}\left(i^{-c_{2} k}+e^{-c_{3} k j}\right)
\end{aligned}
$$

which tends to 1 when $N$ tends to infinity.

Our final result in the section is an extension of Thm. 3.3 to the threshold case. 


\subsection{Theorem}

Let $p \notin X \subseteq \mathbb{R}^{m}, T: \mathbb{R}^{m} \rightarrow \mathbb{R}^{k}$ be a Gaussian random projection, and $d=\min _{x \in X}\|p-x\|$. Then for all $0<\delta<1$ and all $0<\tau<\kappa d$ for some constant $\kappa<1$, we have

$$
\operatorname{Prob}(\operatorname{dist}(T(p), T(X))>\tau)>1-\delta
$$

if $k$ is $O\left(\frac{\log \left(\frac{\lambda X}{\delta}\right)}{\log \left(\frac{d}{\tau}\right)}\right)$.

Proof. For $j=1,2, \ldots$ we construct the sets $X_{j}, S_{j}$ similarly as those in the proof of Thm. 3.3 (where the values of $r_{j}$ and $\varepsilon$ will be defined later). Then we have

$$
\begin{aligned}
\operatorname{Prob}(\exists x \in X \text { s.t }\|T(x)-T(p)\|<\tau) & =\operatorname{Prob}\left(\exists x \in \bigcup_{j=1}^{\infty} X_{j} \text { s.t }\|T(x)-T(p)\|<\tau\right) \\
& \leq \sum_{j=1}^{\infty} \operatorname{Prob}\left(\exists x \in X_{j} \text { s.t }\|T(x)-T(p)\|<\tau\right) .
\end{aligned}
$$

For all $j \geq 1$, we have

$$
\begin{aligned}
& \operatorname{Prob}\left(\exists x \in X_{j} \text { s.t }\|T(x)-T(p)\|<\tau\right) \\
\leq & \operatorname{Prob}\left(\left(\exists x \in X_{j} \text { s.t }\|T(x)-T(p)\|<\tau\right) \wedge \mathcal{E}_{j}^{c}\right)+\operatorname{Prob}\left(\mathcal{E}_{j}\right) \\
\leq & \operatorname{Prob}\left(\exists x \in X_{j}, s \in S_{j} \cap B(x, \varepsilon) \text { s.t }\|T(x)-T(p)\|<\tau \wedge\|T(s)-T(x)\| \leq \varepsilon \sqrt{j}\right)+\operatorname{Prob}\left(\mathcal{E}_{j}\right) \\
\leq & \operatorname{Prob}\left(\exists s \in S_{j} \text { s.t }\|T(s)-T(p)\|<\tau+\varepsilon \sqrt{j}\right)+\lambda_{X}^{\left\lceil\log _{2}\left(\frac{r_{j}+\varepsilon}{\varepsilon}\right)\right\rceil} e^{-c_{1} k j .}
\end{aligned}
$$

Now we choose $\varepsilon=\frac{\tau}{N}$ for some $N>0$ such that $1+\frac{1}{N}<\frac{1}{\kappa}$ and for each $j \geq 1$, we choose $r_{j}=\tau \sqrt{j+1}+(2+j) \varepsilon$. For $j=1$, by the union bound we have

$$
\begin{aligned}
& \operatorname{Prob}\left(\exists s \in S_{1} \text { s.t }\|T(s)-T(p)\| \leq \tau+\varepsilon \sqrt{1}\right) \\
\leq & \lambda_{X}^{\left\lceil\log _{2}\left(\frac{r_{1}+\varepsilon}{\varepsilon}\right)\right\rceil} \operatorname{Prob}\left(\|T(z)\| \leq \frac{\tau+\varepsilon}{d}\right) \quad \text { for an arbitrary } z \in \mathbb{S}^{m-1} \\
= & \lambda_{X}^{\left\lceil\log _{2}(4+N \sqrt{2})\right\rceil} \operatorname{Prob}\left(\|T(z)\| \leq\left(1+\frac{1}{N}\right) \frac{\tau}{d}\right) \quad \text { for an arbitrary } z \in \mathbb{S}^{m-1} \\
< & \lambda_{X}^{\left\lceil\log _{2}(4+N \sqrt{2})\right\rceil}\left(\left(1+\frac{1}{N}\right) \frac{\tau}{d}\right)^{k / 2} \quad \text { by estimation (4) } \\
< & \left(\left(1+\frac{1}{N}\right) \frac{\tau}{d}\right)^{c_{2} k}
\end{aligned}
$$

for some universal constant $c_{2}>0$, as long as $k>\mathcal{C} \log \left(\lambda_{X}\right)$ for some $\mathcal{C}$ large enough.

For $j \geq 2$, we have

$$
\begin{aligned}
& \operatorname{Prob}\left(\exists s \in S_{j} \text { s.t }\|T(s)-T(p)\| \leq \tau+\varepsilon \sqrt{j}\right) \\
\leq & \lambda_{X}^{\left\lceil\log _{2}\left(\frac{r_{j}+\varepsilon}{\varepsilon}\right)\right\rceil} \operatorname{Prob}\left(\|T(z)\| \leq \frac{\tau+\varepsilon \sqrt{j}}{r_{j-1}-\varepsilon}\right) \quad \text { for an arbitrary } z \in \mathbb{S}^{m-1} \\
= & \lambda_{X}^{\left\lceil\log _{2}(3+j+N \sqrt{j+1})\right\rceil} \operatorname{Prob}\left(\|T(z)\| \leq \frac{1}{\sqrt{j}}\right) \quad \text { for an arbitrary } z \in \mathbb{S}^{m-1} \\
< & \lambda_{X}^{\left\lceil\log _{2}(3+j+N \sqrt{j+1})\right\rceil} j^{-k / 2} \quad \text { by estimation (4) } \\
< & j^{-c_{3} k}
\end{aligned}
$$


for some universal constant $c_{3}>0$, as long as $k>\mathcal{C} \log \left(\lambda_{X}\right)$ for some $\mathcal{C}$ large enough.

Similarly, for all $1 \leq j$, we have

$$
\lambda_{X}^{\left\lceil\log _{2}\left(\frac{r_{j}+\varepsilon}{\varepsilon}\right)\right\rceil} e^{-c_{1} k j} \leq e^{-c_{4} k j},
$$

for some universal constant $c_{4}>0$, as long as $k>\mathcal{C} \log \left(\lambda_{X}\right)$ for some $\mathcal{C}$ large enough.

From estimations (6), (17), (8) and by the union bound we have:

$$
\begin{aligned}
\operatorname{Prob}(\operatorname{dist}(T(p), T(X)) \geq \tau) & \geq 1-\sum_{j=1}^{\infty} \operatorname{Prob}\left(\operatorname{dist}\left(T(p), T\left(X_{j}\right)\right)<\tau\right) \\
& \geq 1-\left(\left(1+\frac{1}{N}\right) \frac{\tau}{d}\right)^{c_{2} k}-\sum_{j=2}^{\infty} j^{-c_{3} k}-\sum_{j=1}^{\infty} e^{-c_{4} k j} \\
& \geq 1-\delta \quad \text { for } k=O\left(\frac{\log \left(\frac{\lambda_{X}}{\delta}\right)}{\log \left(\frac{d}{\tau}\right)}\right) \text { large enough. }
\end{aligned}
$$

\section{References}

[1] I. Abraham, Y. Bartal, and O. Neiman. Embedding metric spaces in their intrinsic dimension. In 19th Annual ACM-SIAM Symposium on Discrete Algorithms, page 363372, 2008.

[2] D. Achlioptas. Database-friendly random projections: Johnson-Lindenstrauss with binary coins. Journal of Computer and System Sciences, 66:671-687, 2003.

[3] P. Agarwal, S. Har-Peled, and H. Yu. Embeddings on surfaces, curves, and moving points in Euclidean space. In Proceedings of the 23rd Symposium on Computational Geometry, pages 381-389. ACM, 2007.

[4] S. Dasgupta and A. Gupta. An elementary proof of a theorem by johnson and lindenstrauss. Random Structures and Algorithms, 22:60-65, 2002.

[5] L. Gottlieb and R. Krauthgamer. Proximity algorithms for nearly doubling spaces. SIAM Journal on Discrete Mathematics, 27(4):1759-1769, 2013.

[6] A. Gupta, R. Krauthgamer, and J.R. Lee. Bounded geometries, fractals, and low-distortion embeddings. In IEEE Symposium on Foundations of Computer Science, page 534543. IEEE, 2003.

[7] P. Indyk. Algorithmic applications of low-distortion geometric embeddings. In 42nd Annual Symposium on Foundations of Computer Science, pages 10-33. IEEE, 2001.

[8] P. Indyk and A. Naor. Nearest neighbor preserving embeddings. ACM Transactions on Algorithms, 3(3):Art. 31, 2007. 
[9] W. Johnson and J. Lindenstrauss. Extensions of Lipschitz mappings into a Hilbert space. In G. Hedlund, editor, Conference in Modern Analysis and Probability, volume 26 of Contemporary Mathematics, pages 189-206, Providence, 1984. AMS.

[10] R. Krauthgamer and J.R. Lee. Navigating nets: Simple algorithms for proximity search. In Proceedings of the 15th Annual ACM-SIAM Symposium on Discrete Algorithms, pages 791-801, 2004.

[11] Vu Khac Ky, P.-L. Poirion, and L. Liberti. Using the Johnson-Lindenstrauss lemma in linear and integer programming. Technical Report 07/4985, Optimization Online, 2015.

[12] A. Magen. Dimensionality reductions in $\ell_{2}$ that preserve volumes and distance to affine spaces. Discrete and Computational Geometry, 30(1):139-153, 2007.

[13] J. Matousek. Lectures on Discrete Geometry. Springer, New York, 2002.

[14] A. Mood, F. Graybill, and D. Boes. Introduction to the Theory of Statistics. McGraw-Hill, 1974.

[15] K. Murty and S. Kabadi. Some NP-complete problems in quadratic and nonlinear programming. Mathematical Programming, 39:117-129, 1987.

[16] J.-L. Verger-Gaugry. Covering a ball with smaller equal balls in $\mathbb{R}^{n}$. Discrete Computational Geometry, 33:143-155, 2005. 